\title{
Observation of supercontinuum spiral emission in optical fibers
}

\author{
F. Mangini1, M. Ferraro², M. Zitelli², A. Niang1, A. Tonello ${ }^{3}$, V. Couderc ${ }^{3}$, S. Wabnitz ${ }^{2}$ \\ 1. Department of Information Engineering (DII), University of Brescia, Via Branze 38, 25123 Brescia, Italy. \\ 2. Department of Information Engineering, Electronics and Telecommunications (DIET), \\ Sapienza University of Rome, Via Eudossiana 18, 00184 Rome, Italy. \\ 3. Université de Limoges, XLIM, UMR CNRS 7252, 123 Avenue A. Thomas, 87060 Limoges, France
}

Research on multimode (MM) optical fibers has attracted an increasing interest in recent years, thanks to their application potential [1]. Studies of nonlinear propagation MM fibers are also interesting for a fundamental viewpoint. Thanks to their unique properties, unexpected and yet to be fully understood wave phenomena have been observed in MM fibers. A remarkable example is the appearance of energetic and multi-octave supercontinuum spectra, extending from the visible to the mid-IR, at sufficiently high pumping intensities [2]. It has been shown that pump pulses with $100 \mathrm{ps}$ temporal durations and peak powers around $200 \mathrm{~kW}$ lead to supercontinuum generation in MM fibers with a graded-index (GRIN) core profile [3]. In this work, we reveal that when using femtosecond pump pulses, one has access to a different regime, where the peak power inside the MM fiber may reach and even surpass the critical power $\mathrm{P}_{\mathrm{cr}}$ for catastrophic self-focusing (this is about $10 \mathrm{MW}$ in fusedsilica). When these peak power levels are reached, the propagating light beam undergoes a so-called filamentation phenomenon, leading to the so-called conical emission (CE) [4]. In our experiments, we report for the first time the formation of a supercontinuum spiral emission (SSE), where the spectral components of the output beam are spatially separated in the far-field emitted at the fiber output. We used both a commercial 50/125 GRIN fiber and a 105/125 step-index fiber. Such an unexpected far-field beam shape was obtained by raising the input peak power above the critical threshold $\mathrm{P}_{\mathrm{cr}}$. Our pump laser delivered $120 \mathrm{fs}$ pulses at $1030 \mathrm{~nm}$ with up to $25 \mathrm{MW}$ peak power. The repetition rate was set to $2 \mathrm{kHz}$, and the beam was focused by a $19 \mathrm{~mm}$ lens, producing an $8 \mu \mathrm{m}$ beam waist at the fiber input facet. We show here results with a $2 \mathrm{~cm}$ long multimode standard GRIN and step-index fiber, relative index difference $\Delta=0.0103$ (cladding refractive index is 1.457), and chromatic dispersion $\beta_{2}=-22 \mathrm{ps}^{2} / \mathrm{km}$.

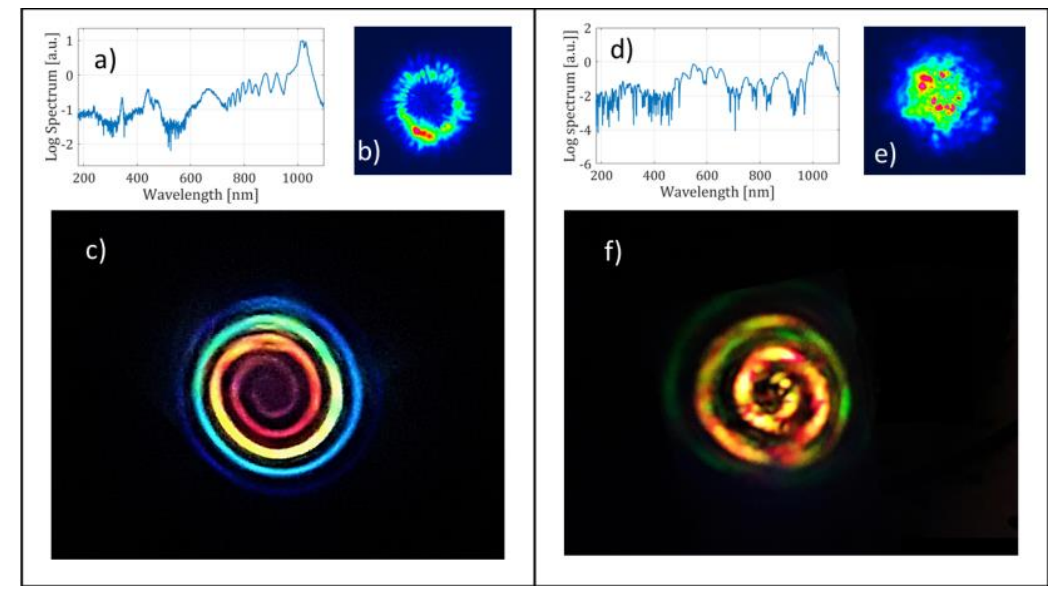

Fig. 1 Experimental results obtained in a 50/125 GRIN fiber (left panel) and Step-index fiber (right panel); a) Output spectrum at $25 \mathrm{MW}$ input peak power (logarithmic scale); b) near field; c) far field.

In Figs.1.a, the output spectrum shows an ultrabroadband distribution, ranging from the UV up to the pump wavelength. The near-field profile at the fiber output shows that the supercontinuum only propagates inside the cladding (see Figs. 1.b). Finally, the far-field SSE is shown in Figs. 1.c. In Fig1 d)-f) we show how the spectrum, the near- and far-field profiles change when the input coupling condition is slightly changed. The spiral-shaped emission is maintained. However, the supercontinuum distribution no longer shows a spatially separated spectral distribution. Moreover, the near-field manifests the typical multimodal speckled profile. A theoretical model able to describe SSE generation is being developed.

This work was supported by the European Research Council (grant No. 740355).

\section{References}

[1] K. Krupa, et al., "Multimode nonlinear fiber optics, a spatiotemporal avenue," APL Photonics 11, 110901 (2019).

[2] L. G. Wright, et al., "Ultrabroadband Dispersive Radiation by Spatiotemporal Oscillation of Multimode Waves" PRL 115, 223902 (2015).

[3] G. Lopez-Galmiche, et al., "Visible supercontinuum generation in a graded index multimode fiber pumped at $1064 \mathrm{~nm}$ " Opt. Lett. 41, 11 (2016).

[4] E. O. Smetanina, et al., "Supercontinuum conical emission accompanying filamentation of a femtosecond laser pulse in fused quartz" J. Opt. Technol. 77, 7 (2010) 